\title{
Quantitative techniques to discriminate petroleum oils using LED-induced fluorescence
}

\author{
V. Rostampour \& M. J. Lynch \\ Curtin University of Technology, Australia
}

\begin{abstract}
In-situ LED-induced fluorescence analysis of oils on water was performed by examining the fluorescence spectrum of various Australian crude oils and some refined oil products. Crude oils and other refined oil were discriminated using non-contact fluorescence-based techniques. The spectral distribution of the fluorescence emission, which contains obvious diagnostic potential, has been used as a quantitative technique to differentiate oil products. The key factors used in these quantitative techniques are; (i) spectral shape, (ii) the wavelength of maximum intensity and (iii) fluorescence spectral width. The first discriminating technique applied is the green-yellow quotient ( $\left.I_{G-Y}=I_{490} / I_{575}\right)$ and the blue-yellow quotient $\left(I_{B-Y}=I_{420} / I_{575}\right)$. The dualwavelength excitation was employed in the laboratory to discriminate crude and refined oils. In this technique, oil slicks (on water) were exposed to two different excitation illumination wavelengths. All oil samples were illuminated by several UV and visible excitation sources. The four crude oils and other petroleum products were easily differentiated by applying the ratio of two fluorescence spectral bands excited by $528 \mathrm{~nm}$ and $405 \mathrm{~nm}$. While theses techniques appear to be sensitive enough to discriminate different crude oils and other refined oil products in the non-contact fluorescence measurement, the selected excitation wavelength range chosen was not the optimum wavelength for fluorescence of crude oil. More studies need to be performed using additional crude oil samples and shorter excitation wavelengths.
\end{abstract}

Keywords: LED-induced fluorescence, oil pollution, crude oil, remote sensing. 


\section{Introduction}

Fluorescence spectra provide valuable information for a variety of purposes for terrestrial and aquatic environments. The major advantages of fluorescence sensing are the possibility of obtaining real-time, low cost, non-contact, and high sensitivity information with high spectral resolution from the target of interest. Fluorescence remote sensing techniques have been used in a variety of environmental studies such as; water pollution [1, 2], turbidity [3], diagnostic of oil pollution [4], chemical pollution [5], pigment composition of phytoplankton [6] and spectral characterization of dissolved organic matter [7].

Fluorescence techniques have been used for decades as tools for the characterization and analysis of hydrocarbon oils. Various aspects of the fluorescence behaviour of mineral oils such as intensity, wavelength distribution, and lifetime have been correlated with petroleum chemical composition and density. Fluorescence is often used to detect the presence of crude oil during drilling operations by observing the fluorescence of the cuttings. Total Scanning Fluorescence (TSF) technique has been used to discriminate drilling additives from crude oils, and to quantify the API gravity (density) of crude oils [8]. In some laboratory-based studies, absolute quantum yields [9, 10] and fluorescence emission spectra of various crude oils have been measured in the visible and the near infrared [9]. Results show the relative dependence of quenching rates on the excitation wavelengths and the chromophore interaction. Fluorescence emission spectra of oil pollution, phytoplankton and dissolved organic mater were registered using a shipboard laser spectrometer at 532 and $266 \mathrm{~nm}$ excitation wavelengths [11].

The Light Detecting And Ranging (LIDAR) technique has been recently used for monitoring oil spills on water surfaces based on the detection of the pollutant fluorescence response and oil characterization. The oil slick thickness is estimated using the suppression of the water Raman signal by an oil film. Laser spectroscopy of mineral oils on the water surface has been reported by Patsayeva et al. [12]. In this research, spectral properties of oil films with various thicknesses on the water surface are studied under controlled laboratory conditions. LIDAR systems operating at excitation wavelengths of 308 and $355 \mathrm{~nm}$ were used the measurement of the fluorescence lifetimes for different mineral oils. Field experiments with airborne fluorescent LIDAR FLS-A were performed to monitor water and land areas for oil transportation and storage. Techniques for real-time data acquisition and processing have been described based on a compiled and systematized library of Laser Induced fluorescence (LIF) spectra of various underlying surfaces [13]. Results confirm that such a system could be implemented in operational use for remote sensing of aquatic and terrestrial targets.

The current work is a preliminary investigation of fluorescence behaviour of hydrocarbons using an LED-induced fluorescensor. The spectral distribution of the fluorescence emission such as spectral shape, maximum intensity and spectral width has been used to differentiate hydrocarbon oils. Two different quantitative techniques have been applied on collected fluorescence spectra to 
detect crude and refined oils in the water environment. Theses techniques performed well and identified hydrocarbon oil slicks on water. Some of refined oils such as kerosene and petroleum spirit indicated no fluorescence response when they were excited by longer UV wavelengths $(365-405 \mathrm{~nm})$. While the rest of the oil samples showed a strong fluorescence emission between $400 \mathrm{~nm}$ and $550 \mathrm{~nm}$, the selected excitation wavelength range chosen was not the optimum wavelength for fluorescence of all petroleum oils. More studies need to be performed using additional crude oil samples and shorter excitation wavelengths.

\section{Experimental}

Four Australian crude oils (Corallina-1, Dockrell-2, Juabert-1 and Enfield-1 and API gravity varying from 33 to 60 and some refined oils (kerosene, regular unleaded petrol, diesel, petroleum spirit and Shell 20W-50 engine oil from) were selected to fluorescence emission measurements. The crude oils selected originate from different locations across the Australia and represent different oil reservoir intervals.

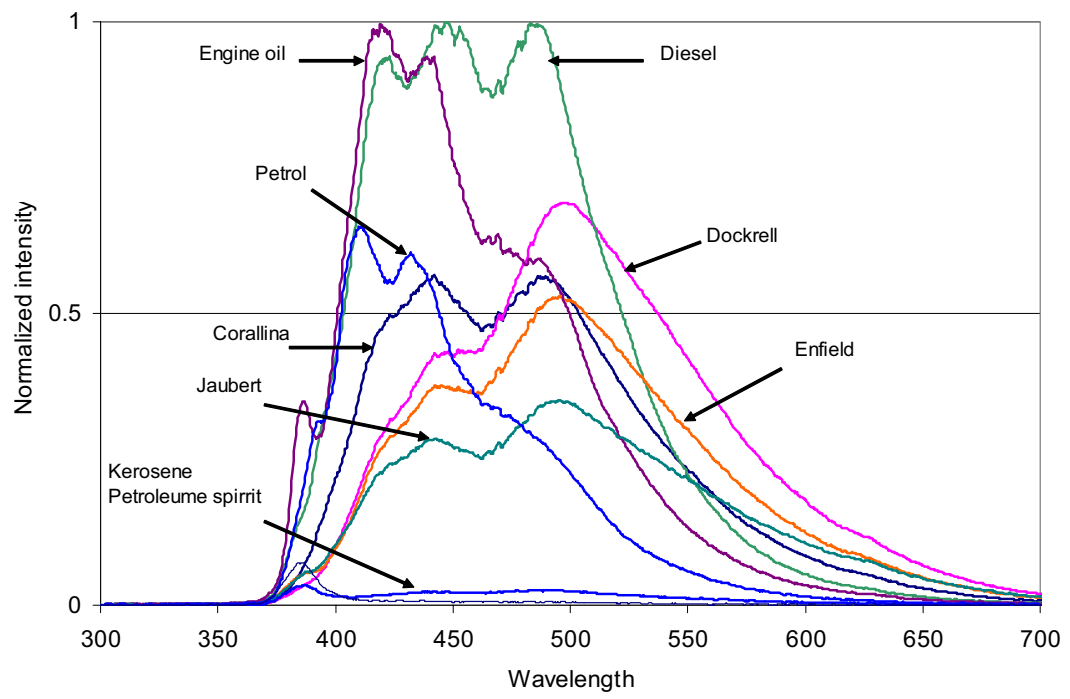

Figure 1: Fluorescence emission spectra of four Australian crude and five refined oils at $385 \mathrm{~nm}$ excitation wavelength.

All oil samples, except kerosene and petroleum spirit, indicated a strong fluorescence emission between $400 \mathrm{~nm}$ and $550 \mathrm{~nm}$ when they were excited by UV wavelength (360-400 nm). Figure 1 shows the fluorescence emission spectra of all oil samples excited by $385 \mathrm{~nm}$ radiation. All fluorescence spectra were normalized to unit maximum intensity. Diesel, petrol and engine oil indicated different shapes of their fluorescence spectra that were composed of 3 bands: one low intensity doublet at $420 \mathrm{~nm}$ and $440 \mathrm{~nm}$, and one at $490 \mathrm{~nm}$. All crude oils 
exhibited reasonable fluorescence response around $560 \mathrm{~nm}$ at the $528 \mathrm{~nm}$ excitation wavelength, while no fluorescence emission was observed for refined oils at this excitation wavelength (figure 2). The only response was the scattered radiation of the excitation source at $528 \mathrm{~nm}$.

A portable LED-induced fluorosensor has been designed to measure fluorescence emission spectra in both the field and laboratory. This fluorosensor system is built around a bundle of monochromatic excitation sources (365 nm to $780 \mathrm{~nm}$ ) and a miniature optical bench. Each excitation source contains a multiLED array. Each multi-LED module is composed of multiple 5-mm diameter ultra bright LEDs (manufactured by ROITHNER LASERTECHNIK GmbH) closely packed into a compact $2-\mathrm{cm}$ diameter aluminium head to achieve a uniform and smooth coverage of illumination. Each excitation source is powered by $4.5 \mathrm{~V}$ DC battery. The long operating time of excitation sources make them suitable for any field operation.

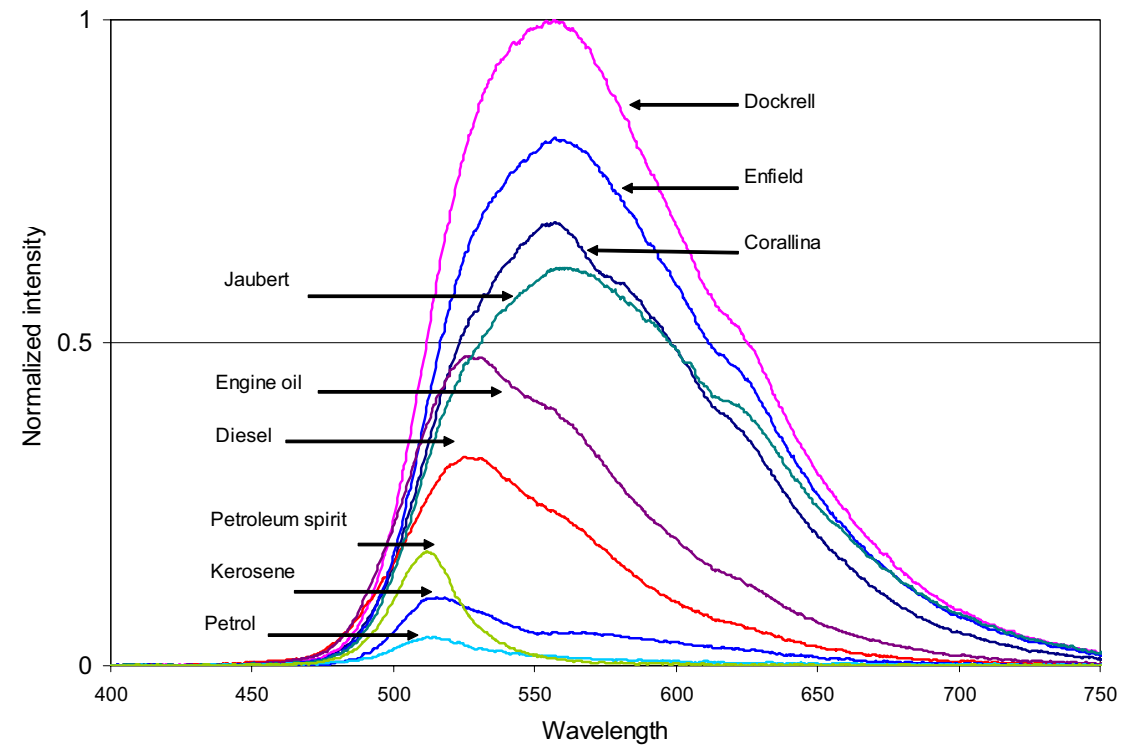

Figure 2: Fluorescence emission spectra of four Australian crude and five refined oils at $528 \mathrm{~nm}$ excitation wavelength.

Fluorescence data from oil samples are recorded over the ultraviolet to shortwave infrared wavelength range using an USB2000-series Spectrometer (Ocean Optics). This miniature fiber optic spectrometer is a high-performance 2048element linear silicon CCD-array detector with sensitivity of 75 photons/count at $400 \mathrm{~nm} ; 41$ photons/count at $600 \mathrm{~nm}$. The spectrometer accepts light energy transmitted through a single-strand optical fiber (SMA 905 to 0.22 numerical apertures) and disperses it via a fixed grating across the linear CCD array detector, which is responsive from 200-1100 nm. Data transfer speed to memory for full scans is $13 \mathrm{~ms}$ with signal-to-noise ratio of 250:1 (at full signal). The 
spectrometer unit is controlled by proprietary OOIBase 32 software and the user may perform spectroscopic measurements such as absorbance, reflectance and emission; control all system parameters; collect data from up to 8 spectrometer channels simultaneously and display the results in a single spectral window; and perform reference monitoring and time-acquisition experiments. In the operational mode the software continually acquires data from the spectrometer. An integration time needs to be set and this affects the collected intensity. In order to be able to easily compare spectra they should be taken at the same integration time [14].

The system is capable of real-time measurements, on-the-spot analysis of released pollutants such as hydrocarbons and eliminates costly sample preparation and analytical procedures. In addition, the USB cable connects the spectrometer to the computer. Accordingly, no additional external power supply is required.

\section{Results and discussion}

Oil samples were poured onto a water surface housed in a black-coated container. LEDs and fiber optics were placed approximately five $\mathrm{cm}$ away from the samples. The exciting light beam was incident the oil slick surface from above and the LED-induced fluorescence signals were collected through a fiber optic cable. A wide range of excitation wavelengths in the UV (365 to $395 \mathrm{~nm}$ ), visible $(405 \mathrm{~nm}$ to $700 \mathrm{~nm})$ and near Infrared $(780 \mathrm{~nm})$ have been used. The fluorescence emission was recorded by time integrating 10 scans for each sample. Replicate values were averaged.

The excitation source configuration for illumination of the samples is an important consideration. The BRDF of oil surface is strongly affected by the excitation source angle of incidence and the view direction (fiber optic detection angle) geometry. A smooth surface of oil films show the highest possible reflectance at the specular angle and the spectrometer can be saturated for this geometry.

Quotient measurements were made using ratio recording of two fluorescence emission wavelengths. The method first involved an investigation to select key wavelengths in the UV-Visible-Near Infrared spectral interval. Three dominant wavelengths $(420,490$, and $575 \mathrm{~nm})$ were identified. Most of the hydrocarbon oils were classified with the blue-yellow quotient $\left(I_{B-Y}=I_{420} / I_{575}\right)$ and the greenyellow quotient $\left(I_{G-Y}=I_{490} / I_{575}\right)$, where $\mathrm{I}$ is the measured fluorescence relative intensity. The blue-yellow quotient and the green-yellow quotient for various crude and refined oils at the $375 \mathrm{~nm}, 385 \mathrm{~nm}, 395 \mathrm{~nm}$ and $405 \mathrm{~nm}$ excitation wavelengths are shown in figure 3. In all cases, values were significantly lower for crude oils than for refined oils for the green-yellow quotient. The same behaviour was observed for the blue-yellow quotient; however, there was some variability in the quotient value of refined oils. This method appears to be sensitive to differentiate crude oils from refined oils in the field environment. 

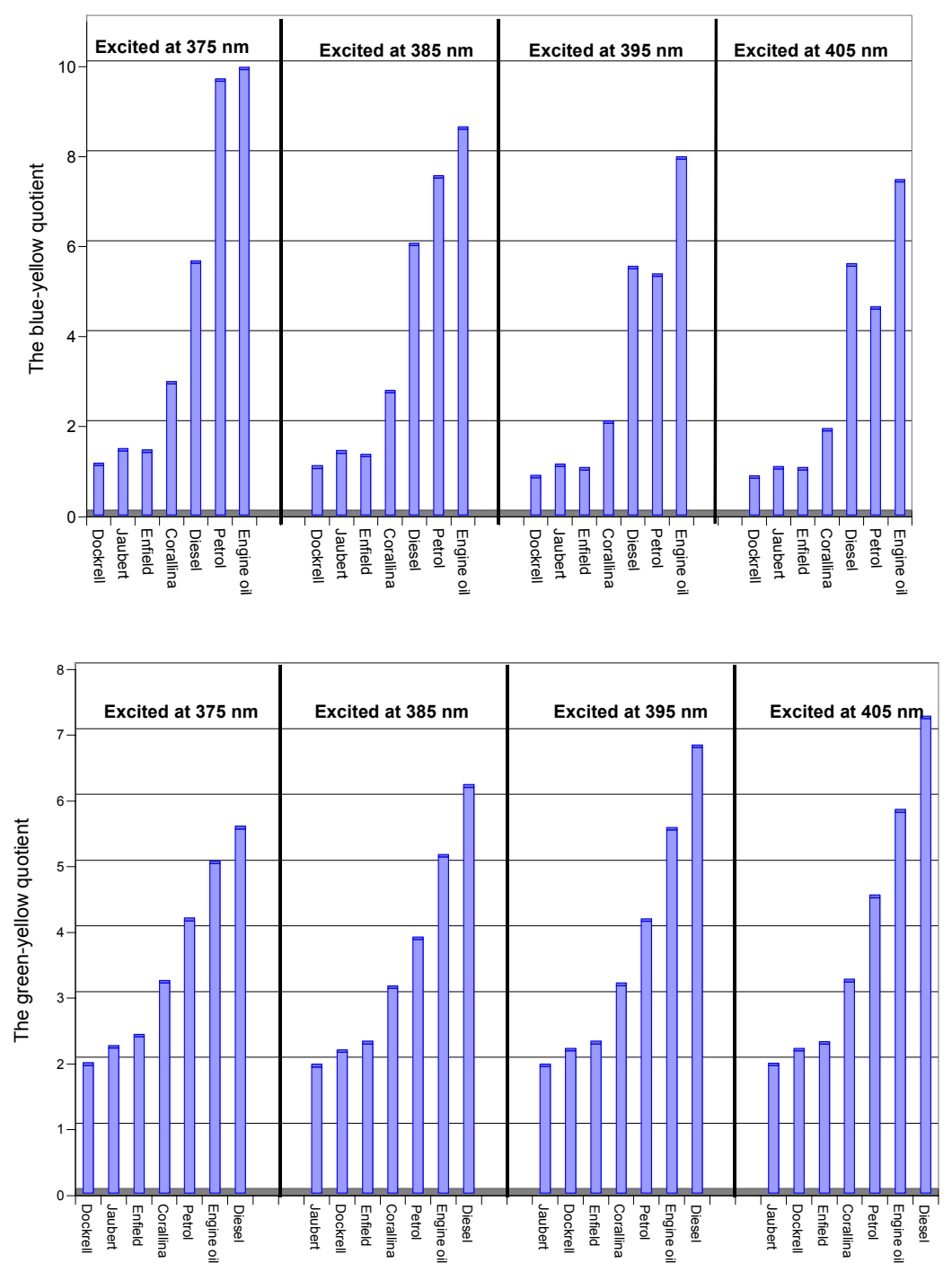

Figure 3: The blue-yellow quotient and the green-yellow quotient of various hydrocarbon oils at the $375 \mathrm{~nm}, 385 \mathrm{~nm}, 395 \mathrm{~nm}$ and $405 \mathrm{~nm}$ excitation wavelengths.

In some cases, it could be difficult to differentiate between various petroleum oils using fluorescence spectra alone or visual inspection when using a single excitation source, since some hydrocarbons have similar fluorescence spectral shapes. However, it is possible to differentiate between oils when the fluorescence intensity is reasonably higher than others. In a similar manner, a technique such as the dual-wavelength excitation fluorescence ratio may be used 
to differentiate between crude and refined oils. The fluorescence emission spectra at $405 \mathrm{~nm}$ excitation wavelength divided by the fluorescence emission spectra at $528 \mathrm{~nm}$ excitation wavelength was termed the dual-wavelength excitation fluorescence ratio. The steady state fluorescence ratio of dualwavelength excitation versus wavelength, at similar temperatures and film thicknesses, are shown in figure 4 for various crude and refined oils. According to figure 4, the ratio increase with rising density of hydrocarbons. Engine oil and two heavy crude oils, Enfield and Jaubert, indicate the highest ratio, while petrol, diesel and kerosene show the lowest ratio, respectively.

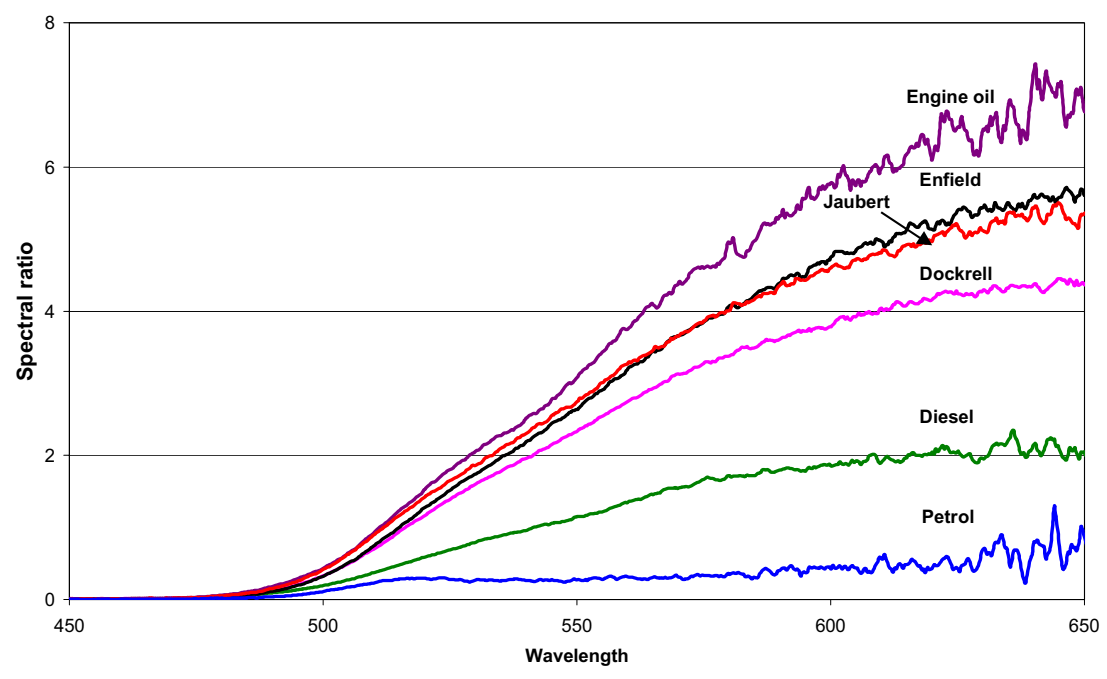

Figure 4: Ratio of the fluorescence emission spectra at $528 \mathrm{~nm}$ and $405 \mathrm{~nm}$ excitation wavelength for various hydrocarbon oils.

\section{Conclusion}

The thin fluorescence spectrum of four Australian crude oils and some refined oils were examined using in-situ LED-induced fluorescence analysis using wide range of excitation wavelengths from the UV to the near infrared. The spectral distribution of the fluorescence emission, which contains obvious diagnostic potential, has been used as a quantitative technique to differentiate oil products.

The objective of this research is to validate quantitative techniques to detect crude and refined oils in the marine environment. The blue-yellow $\left(I_{B-Y}=I_{42} / I_{575}\right)$ and the green-yellow $\left(I_{G-Y}=I_{49} / I_{575}\right)$ quotient have been developed and applied as fluorescence-based method to identify crude from refined oils. In addition, the dual-wavelength excitation fluorescence ratio can differentiate between crude and refined oils. Although these techniques performed reasonably well in the laboratory environment, they need to be validated in field condition using additional crude oil samples and excitation wavelengths. 


\section{Acknowledgement}

The contribution of crude oil supplies to the project by Woodside and Geotechnical Services PTY LTD, Western Australia is gratefully acknowledged.

\section{References}

[1] Baker, A., Inverarity, R., Charlton, M.E. and Richmond, S., Detecting river pollution using fluorescence spectrophotometry: case studies from the Ouseburn, NE England. Environmental Pollution, 124, 57-70 2003.

[2] Reynolds, D.M. and Ahmad, S.R., Rapid and direct determination of wastewater BOD values using a fluorescence technique, Water Research 31, pp. 2012-2018, 1997.

[3] Hoge F.E., Swift R. N., Airborne detection of ocean turbidity cell structure using depth-resolved laser induced water Raman backscatter, Applied Optics, 22:

[4] Dolenko T. A., Fadeev V. V., Gerdova I. V., Dolenko S. A., Reuter R., Fluorescence diagnostics of oil pollution in coastal marine waters using artificial neural networks, Applied Optics, 41, 5155-5166, 2002.

[5] Dudelzak A.E., Babichenko S.M., Poryvkina L.V., Saar K. U., Total luminescent spectroscopy for remote laser diagnostics of natural water conditions, Applied Optics, v. 30, No. 4, 453-458,1991. 3778-3786, 1983.

[6] Kaitala S., Babichenko S., Poryvkina L., and Leeben A., Fluorescent Analysis of Pigment Composition of Natural Phytoplankton, Marine Technology Society Journal, 28: 50 - 58, 1994.

[7] Poryvkina, L.V., Babichenko, S.M. and Lapimaa, J.J., Spectral variability of humus substance in marine ecosystems, Ambio, 21:465-467, 1992.

[8] Fder A.G., Iwanski P. and Montanari L., Light Emissions from Oil: A valuable Information source for petroleum analysis, TPoint, \#2, pp.9-14, 2004.

[9] Downare, Taggart D., Mullins, Oliver C., Visible and Near-Infrared Fluorescence of Crude Oils, Applied Spectroscopy, Volume 49, Number 6, pp. 754-764(11), 1995.

[10] Ralston, Corie Y., Wu, Xu, Mullins, Oliver C., Quantum Yields of Crude Oils, Applied Spectroscopy, Volume 50, Number 12, pp. 1563-1568(6), 1996.

[11] Chubarov V. V. and Fadeev V. V., Ecological monitoring in the Caspian Sea (mouth zone of the river Volga) with a shipboard laser spectrometer, EARSeL eProceedings 3, 2004.

[12] Patsayeva S., Yuzhakov1 V., Varlamov V., Barbini R., Fantoni R., Frassanito C. and Palucci A., Laser spectroscopy of mineral oils on the water surface, Proceedings of EARSeL-SIG-Workshop, 2000.

[13] Babichenko S., Dudelzak A. and Poryvkina L., Laser remote sensing of coastal and terrestrial. Pollution by fls-lidar, EARSeL eProceedings 3, 2004.

[14] Ocean optics, OOIBase32 spectrometer operating software manual. 2000. 